\title{
Review of Introductory Tests to in Vivo Evaluation, Prototypes Assembling and Anatomical Position Studies after Five Years
}

\author{
Eduardo GP Bock* \\ Federal Institute of Technology, Sao Paulo, Brazil
}

Received: October 17, 2016; Accepted: October 20, 2016; Published: November 03, 2016

*Corresponding author: Eduardo GP Bock, Federal Institute of Technology, Sao Paulo,Brazil, E-mail: eduardobock@gmail.com

\begin{abstract}
A Mechanically Assisted Circulatory Support device called Implantable Centrifugal Blood Pump is been developed in Federal Institute of Technology in Sao Paulo, Brazil. The equipment has original features for application as a Ventricle Assist Device (VAD) during advanced stages of Heart Failure. This pump is part of a multicenter and international study started in 2006 with objective to offer simple, affordable and reliable devices to developing countries. Previous computational fluid dynamics investigations were performed in 2008 followed by prototyping and in vitro tests. Also, previous blood tests for assessment of Hemolysis shown mean NIH results of $0.0054 \pm$ $2.46 \times 10^{-3} \mathrm{mg} / 100 \mathrm{~L}$ (at $5 \mathrm{~L} / \mathrm{min}$ and $100 \mathrm{mmHg}$ ) in 2011. In order to precede in vivo evaluation, measurements of magnetic coupling interference and enhancements of actuator control were necessary. Methodology was based in study of two different work situations (1 and 2) studied with two different types of motor (A and B). Situation 1 is when the rotor of pump is closest to the motor and Situation 2 its opposite. Torque and mechanical power were collected with a dynamometer $(80$ g.cm) then plotted and compared for two situations and both motors. Since the first publication of introductory tests results in 2011, new findings were achieved and they are described in this paper. The results showed that motor A has better mechanical behavior and less influence of coupling. Results for Situation 1 showed that it is more often under magnetic coupling influence than Situation 2. The studies lead the conclusion that motor $A$ is the best option for in vivo studies since it has less influence of magnetic coupling in both situations. New computational fluid dynamics simulations will be conducted in order to optimize geometry of pump and actuator.
\end{abstract}

\section{Introduction}

A Mechanically Assisted Circulatory Support (MACS) device called Implantable Centrifugal Blood Pump (ICBP) is been developed in Federal Institute of Technology in Sao Paulo, Brazil [1].

ICBP has original features for MACS application. The prescription, therapies and results of this type of medical device are described in the Interagency Registry for Mechanically Assisted Circulatory Support (INTERMACS) [2].

ICBP was designed in 2006 to long term application of
Ventricle Assist Device (VAD) during advanced stages of Heart Failure as Bridge to Transplant (BTT) and Bridge to Recovery (BTR) and Destination Therapy (DT), Figure 1.

This pump is part of a multicenter and international study started in 2006 with objective to offer simple, affordable and reliable devices to developing countries. The motor controller was designed to drive the actuator (ML4426, MicroLinear, San Jose). Previous computational fluid dynamics investigations were performed in 2008 followed by prototyping and in vitro tests [3].

The design methodologies of ICBP followed the "step-bystep" procedures proposed by Nose (1998) with previous computational simulations and introductory tests. Numerical electromagnetic simulation and the Brushless Direct Current (BLDC) motor optimizations were performed with software Flux 3D followed by prototyping and in vitro tests [4]. Figure 2.

Also, previous blood tests for assessment of Hemolysis shown mean NIH results of $0.0054 \pm 2.46 \times 10-3 \mathrm{mg} / 100 \mathrm{~L}$ (at $5 \mathrm{~L} / \mathrm{min}$ and $100 \mathrm{mmHg}$ ).

In order to precede in vivo test, measurements of magnetic coupling interference and enhancements of actuator control were necessary.

Since the first publication of introductory tests results in 2011, new findings were achieved and they are described in this review paper [3].

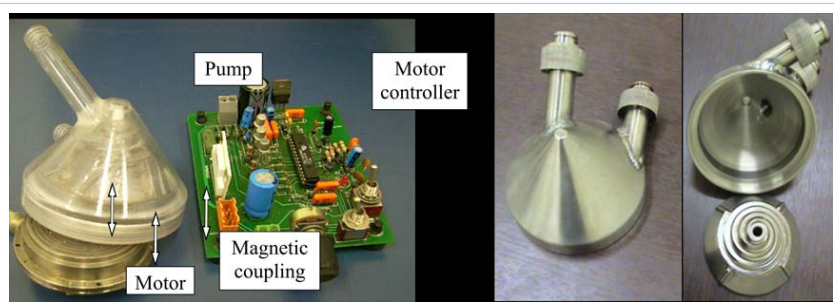

Figure 1: Pictures of ICBP prototypes (a) Motor, magnetic coupling, controller and Pump machined in acrylic for in vitro performance tests; (b) Pump made with titanium and rotor disassembled 


\section{Method}

Methodology was based in the study of two different work situations of the ceramic polymeric ICBP pivot bearings ( 1 and 2). Situation 1 is when the lower pivot bearing has contact between the ceramic pivot shaft and the polymeric female bearing. In contrast, situation 2 is when the upper pivot bearing has contact between the ceramic pivot shaft and the polymeric female bearing, as seen on Figure 3.

The two different work situations were studied and compared with two different types of motor (A and B) with 3 pairs of windings and 4 permanent magnets or with 12 pairs of windings and 8 permanent magnets, as seen on Figure 4.

Situation 1 is when the rotor of pump is closest to the motor and the electromagnetic field can has more influence, but situation 2 is the opposite. In both cases, situations were simulated with spacers located between pump and actuator.

The experimental apparatus, Figure 5, was assembled as a "mock loop circuit" $[5,6]$ in order to evaluate the hydrodynamically performance of the pumps.

The circuit was composed by 2 liters acrylic reservoir, 3/8" silicone tubes to connect ICBP and reservoir, aqueous solution with $37 \%$ glycerin in $25^{\circ} \mathrm{C}$ in order to simulate the blood viscosity $[7,8]$.

Pressure was measured with a monitor (DX 2020, Dixtal Biomedica, Sao Paulo), flow was measured with Flow meter (HT110 Transonic, New York, EUA), power supply and oscilloscope (MO 1227, Minipa, Shangai)

Torque and mechanical power were collected in three different speeds 1500 RPM, 2000 RPM and 2500 RPM, with a (80 g.cm) dynamometer (HD 100-7, Magtrol, Buffalo) then plotted and compared for two situations and both motors, Figure 6 [3].

\section{Results}

The results presented in 2011 showed that motor $\mathrm{A}$ had better mechanical behavior and less influence of coupling distance than motor B. Results for Situation 1 showed that it was more often under magnetic coupling influence than Situation 2, as seen on Figure 7.

The curves of the two different situations were plotted from

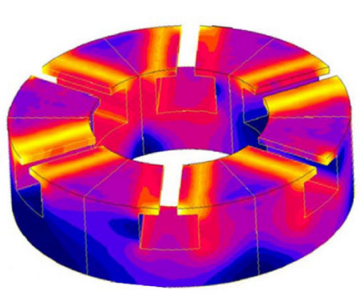

(a)

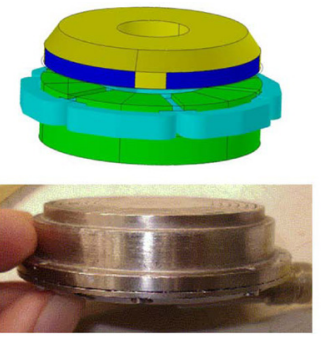

(b)
Figure 2: Computational numerical simulations and ICBP actuator (a) results in FLUX 3D, and (b) titanium prototype

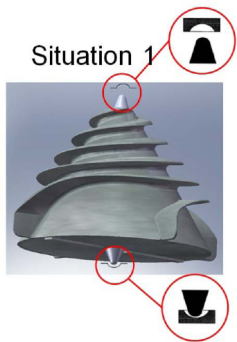

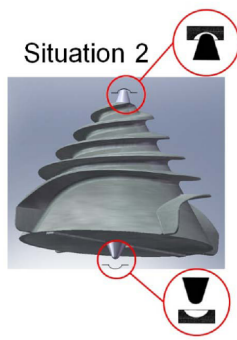

Figure 3: The two different work situations of the ceramic polymeric ICBP pivot bearings studied (a) with contact in lower pivot bearing and (b) with contact in upper pivot bearing

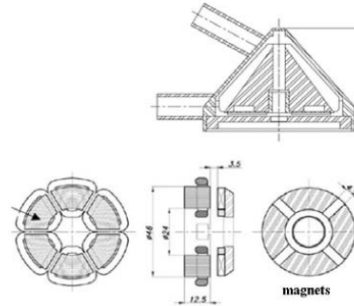

(a)
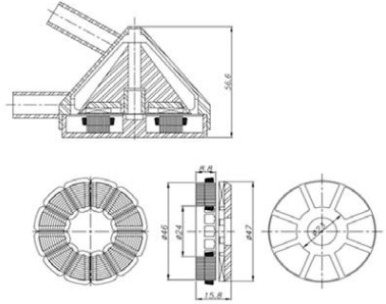

(b)
Figure 4: The two different types of motor: (a) with 3 pairs of windings and 4 permanent magnets and (b) with 12 pairs of windings and 8 permanent magnets

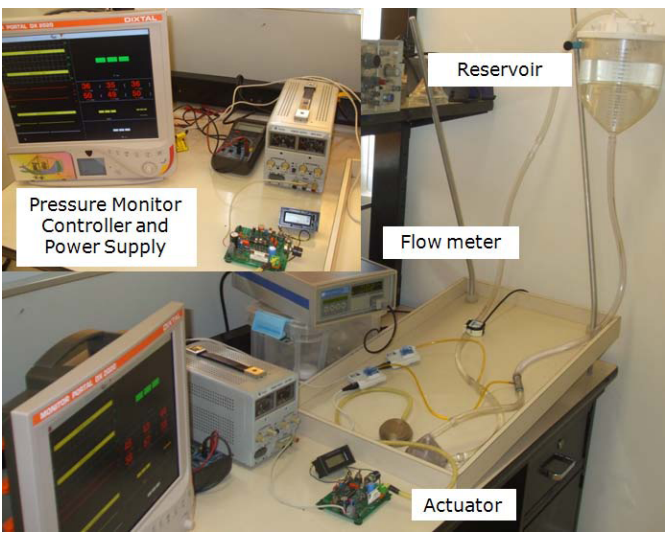

Figure 5: The "mock loop circuit" assembled to evaluate the hydrodynamically performance of the pumps

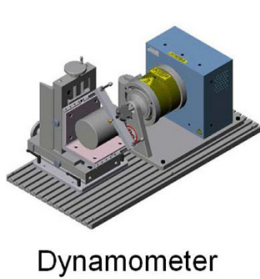

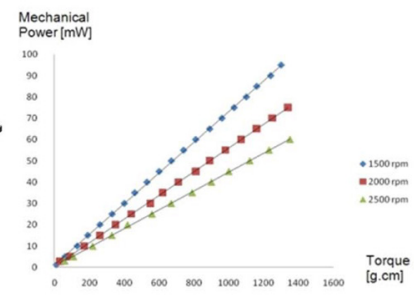

Figure 6: Torque and mechanical power measurements (a) Dynamometer and (b) three different speed plots 


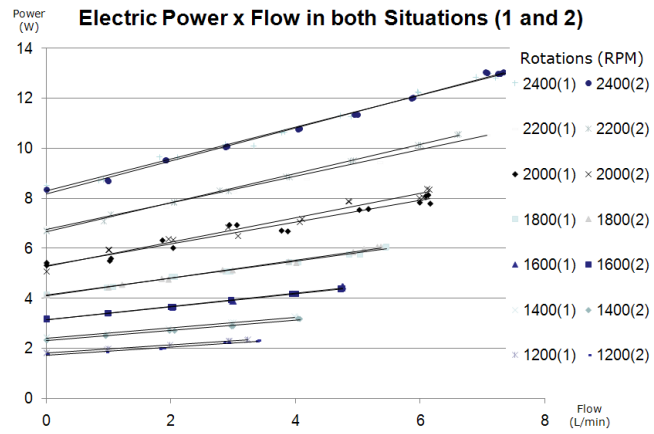

Figure 7: The plotted curves for seven speeds showing electric power versus flow in situations 1 and 2

speed rotations of 1200 RPM to 2400 RPM. It was possible to observe a slight tendency in variation of curves, especially in 1800 RPM and 2000 RPM but other curves were considered equals. The operational range of ICBP can vary but this increase in power consumption was not observed as a problem during that time, published only in recent works [9].

\section{Conclusion}

In the original paper, the study's results lead the conclusion that motor A was the best option for in vivo studies since it had less influence of magnetic coupling in both situations. It is a strong argument to decide which model is more reliable and durable.

New computational fluid dynamics simulations will be conducted in order to optimize geometry of pump and actuator. New models of actuators and pumps are already been modeled.

After five years, this review paper can affirm that is still no consensus of the best geometry and topography of actuator available for the ICBP device designed in Brazil. Our group is now working in the Laboratory of Bioengineering and Biomaterials of the Federal Institute of Technology in Sao Paulo, designing new pumps, new biomaterials for the pivot bearings and working on improve the reliability and the durability of VAD.

\section{Acknowledgments}

The authors are grateful to FAPESP, CAPES and CNPq for partially supporting this research.

\section{References}

1. Bock E, Ribeiro A, Silva M, Antunes P, Fonseca J, Legendre D, et al. New centrifugal blood pump with dual impeller and double pivot bearing system: wear evaluation in bearing system, performance tests, and preliminary hemolysis tests. Artif Organs. 2008;32(4):329-333. doi: 10.1111/j.1525-1594.2008.00550.x.

2. Kirklin JK, Naftel DC, Pagani FD, Kormos RL, Stevenson LW, Blume ED, et al. Seventh INTERMACS annual report: 15,000 patients and counting. J Heart Lung Transplant. 2015;34(12):1495-1504. doi: 10.1016/j.healun.2015.10.003

3. Bock E, Andrade A, Dinkhuysen J, Arruda C, Fonseca J, Leme J, et al. Introductory Tests to In Vivo Evaluation: Magnetic Coupling Influence in Motor Controller. ASAIO J. 2011;57(5):462-465. doi: 10.1097/ MAT.0b013e31823005dc.

4. Nosé Y. Design and development strategy for the rotary blood pump. Artif Organs. 1998;22:438-446.

5. Ohara Y, Sakuma I, Makinouchi K, Damm G, Glueck J, Mizuguchi K, et al. Baylor gyro pump: a completely seal-less centrifugal pump aiming for long-term circulatory support. Artif Organs. 1993;17(7):599-604.

6. Watanabe K, Ichikawa S, Asai T, Motomura T, Hata A, Ito S, et al Centrifugal blood pump with a hydraulically-levitated impeller for a permanently implantable biventricular assist device. Artif Organs. $2004 ; 28(6): 556-563$.

7. Legendre D, Antunes P, Bock E, Andrade A, Biscegli JF, Ortiz JP. Computational fluid dynamics investigation of a centrifugal blood pump. Artif Organs. 2008;32(4):342-348. doi: 10.1111/j.15251594.2008.00552.x

8. Fonseca J, Andrade A, Nicolosi DE, Biscegli JF, Legendre D, Bock E, et al. A new technique to control brushless motor for blood pump application. Artif Organs. 2008;32(4):355-359. doi: 10.1111/j.15251594.2008.00554.x.

9. Bock E, Leao T, Fonseca J, Andrade A. Left Ventricle Failure and Blood Flow Estimation for Centrifugal Blood Pumps. J Mech Eng Autom. 2016;6:162-166. doi: 10.17265/2159-5275/2016.03.007. 